\title{
Expression of ELF5 in endometrial carcinoma tissues and its clinical significance
}

\author{
$\mathrm{HE} \mathrm{YANG}^{1}$ and HONGCHAO YAN ${ }^{2}$ \\ ${ }^{1}$ Graduate School of Xuzhou Medical University, Xuzhou, Jiangsu 221004; ${ }^{2}$ Department of Obstetrics and \\ Gynecology, The Affiliated Hospital of Xuzhou Medical University, Xuzhou, Jiangsu 221006, P.R. China
}

Received September 4, 2017; Accepted May 22, 2018

DOI: $10.3892 / \mathrm{ol} .2018 .9093$

\begin{abstract}
Expression of E74-like factor 5 (ELF5) in endometrial carcinoma tissues and its clinical significance were investigated. Eighty-four endometrial carcinoma tissues, 30 cases of atypical hyperplasia of endometrium and 30 cases of normal endometrial tissues were selected. Immunohistochemical method was utilized to detect the expression of ELF5 in different endometrial tissues. Moreover, its correlation with clinical pathological indexes of patients with endometrial carcinoma was analyzed. The postoperative follow-up was conducted in all the patients with endometrial carcinoma until June 30th, 2017. The Kaplan-Meier method was used for survival analysis so as to analyze the association of ELF5 expression level with clinical pathological indexes; Cox's proportional hazards regression model was utilized for univariate and multivariate analyses to screen independent risk factors for prognosis of endometrial carcinoma. In normal endometrial tissues, atypical hyperplasia and endometrial carcinoma tissues, the positive expression rates of ELF5 showed a decreased tendency $(\mathrm{P}=0.016)$. The positive expression rate of ELF5 in endometrial carcinoma tissues was lower in comparison to normal endometrial tissues $(\mathrm{P}=0.016)$. The expression of ELF5 was in accordance with the International Federation of Gynecology and Obstetrics (FIGO) staging of endometrial carcinoma $(\mathrm{P}<0.05)$, pathological grading $(\mathrm{P}<0.05)$, pathological typing $(\mathrm{P}=0.001)$, state of lymph node metastasis $(\mathrm{P}<0.05)$ and depth of myometrial invasion $(\mathrm{P}<0.05)$. Kaplan-Meier method for survival analysis showed that the average survival time of patients with negative ELF5 expression was shorter in comparison to the patients with positive expression $(\mathrm{P}=0.004)$. FIGO staging $(\mathrm{P}=0.004)$, pathological grading $(\mathrm{P}=0.048)$, depth of myometrial invasion
\end{abstract}

Correspondence to: Dr Hongchao Yan, Department of Obstetrics and Gynecology, The Affiliated Hospital of Xuzhou Medical University, 99 Huaihai West Road, Xuzhou, Jiangsu 221006, P.R. China

E-mail: 13952158787@163.com

Key words: endometrial carcinoma, suppressor gene, ELF5 gene, immunohistochemistry, survival analysis
$(\mathrm{P}=0.024)$ and lymph node metastasis $(\mathrm{P}=0.020)$ were related to the prognosis of patients with endometrial carcinoma, The univariate Cox's regression model analysis indicated that FIGO staging $(\mathrm{P}=0.010)$, pathological grading $(\mathrm{P}=0.040)$, depth of myometrial invasion $(\mathrm{P}=0.037)$, lymph node metastasis $(\mathrm{P}=0.029)$ and ELF5 $(\mathrm{P}=0.010)$ were associated with the prognosis of patients with endometrial carcinoma. Further, multivariate analysis revealed that ELF5 was an independent risk factor for prognosis of patients with endometrial carcinoma $(\mathrm{P}=0.035)$. The expression of ELF5 has a correlation with the occurrence, development and prognosis of endometrial carcinoma.

\section{Introduction}

Endometrial carcinoma is the most common type of gynecological malignant tumor, ranking fourth in female cancers $(1,2)$. The detection rate of endometrial carcinoma is on the rise among young women who have a demand for childbearing. The epidemiologic investigation has confirmed that approximately $1.6 \%$ of patients with endometrial carcinoma are aged 20-34 years, and 6.1\% are aged 35-44 years (3). Furthermore, the prognosis of patients with early endometrial carcinoma is quite good, after surgical treatments and adjunctive therapies. However, patients usually get late diagnosis due to lack of effective and early diagnostic methods (4). There are multiple factors affecting therapeutic treatment of endometrial carcinoma, such as clinical factors (surgical-pathologic staging, depth of myometrial invasion and adjunctive therapy) and biological factors (steroid receptors, growth factors, oncogenes and suppressor genes) $(5,6)$. Recently, screening of biological indexes and gene therapies for endometrial carcinoma is becoming an active research field.

E74-like factor 5 (ELF5) (also known as ESE2), is a member of the E-twenty-six (ETS) transcription factor family which regulates cell proliferation, differentiation and apoptosis. Further, it has epithelial specificity and plays an important regulatory role in placenta, lung airway and mammary gland (7-10). A recent study by Chakrabarti et al (11) showed that ELF5 has potential effects of suppressor genes in normal mammary gland. Loss of ELF5 expression might lead to proliferation of adult mammary stem cells, increasing the risk of breast cancer. As for ovarian cancer, earlier studies of the above research group discovered that the expression of 
ELF5 mRNA showed decrease in epithelial ovarian cancer tissues. The loss of expression affected the occurrence and development of the disease. It might inhibit the invasion and metastasis of ovarian cancer cells by regulation of matrix metalloproteinase-2 (MMP-2) and MMP-9. In this way, it accelerates cell cycle and apoptosis by increasing the expressions of P21 and caspase-3 in the human ovarian cancer tissues (12-14). However, in endometrial carcinoma, the effects of ELF5 have not been fully revealed. Therefore, the present study was planned to explore the expression of ELF5 in endometrial carcinoma and its clinical significance. The study would provide new targets and ideas for diagnosis, prognosis and treatment of endometrial carcinoma.

\section{Materials and methods}

Study subjects. All the specimens were excised from the patients who received surgical treatments in the Department of Obstetrics and Gynecology, The Affiliated Hospital of Xuzhou Medical University (Jiangsu, China) from January 2010 to December 2014. The Ethics Committee of the hospital approved the study, and signed informed consent was obtained from all the patients. Inclusion criteria for endometrial carcinoma group were as follows: Patients with endometrial carcinoma having complete clinical and pathological data along with postoperative follow-up. The exclusion criteria were: patients complicated with other systemic malignant tumors or metastatic carcinomas from tumors of other organs in the reproductive system (including synchronous primary carcinomas) as well as patients who received chemotherapy, radiotherapy or endocrine therapy as the first line of treatment were excluded.

Study design. The present study was divided into three groups $v i z$. endometrial carcinoma group, atypical hyperplasia group and a normal endometrial tissue group.

i) Endometrial carcinoma group: this group included paraffin blocks of tissues excised from 84 patients, which were confirmed surgically and pathologically for endometrial carcinoma. The patients were aged 38-70 years, with an average age of 54.61 years. According to the surgical-pathologic staging [International Federation of Gynecology and Obstetrics (FIGO), 2009] the subjects were classified into different stages. There were 54 cases in stage I, 10 cases in stage II and 20 cases in stage III+IV. Further, there were 52 cases with the depths of myometrial invasion $\leq 1 / 2$ and 32 cases $>1 / 2$. Forty-one cases were accompanied with lymph node metastasis and 26 cases without lymph node metastasis (with a total of 67 cases undergoing lymphadenectomy). The pathological typing was composed of 68 cases with endometrioid adenocarcinoma and 16 cases with non-endometrioid adenocarcinoma. The pathological grading of endometrial adenocarcinoma showed 50 cases of G1, 8 cases of G2 and 10 cases of G3; 15 patients had menstruation and 69 patients had no menstruation.

ii) Atypical hyperplasia group: this group included paraffin blocks of 30 patients who received surgical treatments due to dysfunctional uterine bleeding. These patients were confirmed pathologically for atypical hyperplasia and archived in the same period. The patients were aged 40-74 years, with an average age of 45.93 years.
Table I. ELF5 expression in different endometrial tissues.

\begin{tabular}{lccccc}
\hline Group & $\mathrm{n}$ & $\begin{array}{c}\text { Positive } \\
\text { (case) }\end{array}$ & $\begin{array}{c}\text { Positive } \\
(\%)\end{array}$ & $\chi^{2}$ & P-value \\
\hline $\begin{array}{l}\text { Normal group } \\
\begin{array}{l}\text { Atypical } \\
\text { hyperplasia }\end{array}\end{array}$ & 30 & 28 & 93.33 & $5.787^{\mathrm{a}}$ & $0.016^{\mathrm{a}}$ \\
$\begin{array}{l}\text { Endometrial } \\
\text { carcinoma }\end{array}$ & 84 & 58 & 69.05 & $1.614^{\mathrm{c}}$ & $0.204^{\mathrm{c}}$ \\
\hline
\end{tabular}

andicates comparisons between normal and endometrial carcinoma groups; 'indicates comparisons between normal and atypical hyperplasia groups; cindicates comparisons between atypical hyperplasia and endometrial carcinoma groups. ELF5, E74-like factor 5.

iii) Normal endometrial tissue group: this group included paraffin blocks of 30 patients who received surgical treatments due to hysteromyoma, but were pathologically confirmed as normal endometrial tissues. Subsequently they were also archived in the same period. The patients were aged 37-51 years, with an average age of 49.73 years.

Detection of ELF5 protein expression in tissues. All the specimens were fixed with $4 \%$ neutral formalin, embedded in paraffin and serially sliced to thickness of $4 \mu \mathrm{m}$. The slices were dipped and were dewaxed in xylene and gradient ethanol and $\mathrm{H}_{2} \mathrm{O}_{2}(3 \%)$ was then added and were incubated at room temperature for $10 \mathrm{~min}$, in order to eliminate the activity of endogenous peroxidase. This was followed by execution of antigen retrieval with citrate buffer under high pressure. ELF5 antibody was dripped and incubated at $4{ }^{\circ} \mathrm{C}$ overnight. The hypersensitive two-step rabbit detection kit and DAB kit were utilized for color development. The specimens were counterstained, conventionally dehydrated, cleared, mounted with neutral balsam and then observed under a microscope for the staining results. The ELF5 antibody (bs-14564R) was procured from Beijing Bioss Biological Technology Co., Ltd. (Beijing, China), and the hypersensitive two-step rabbit detection kit (PV-9001) and DAB kit (ZLI9018) were purchased from Beijing Zhongshan Goldenbridge Biotechnology Co., Ltd. (Beijing, China).

Staining analyses. The staining results were analyzed via double-blind reviewing method. Two experienced physicians reviewed the images. Ten high-power fields with 100 cells each were randomly selected using semi-quantitative method. The cells were given points according to the percentage of positive cells in each high-power field: percentage of positive cells $<5 \%$, 0 point; $5-25 \%, 1$ point; $26-50 \%, 2$ points; $51-75 \%, 3$ points; $>75 \%, 4$ points. Furthermore, scores were recorded according to the color of the nuclei: no coloring, 0 point; light yellow, 1 point; yellow, 2 points; and brownish yellow, 3 points. The product of the scores of the two scoring systems was regarded as the total score: 0 point, negative; $1-4$ points, weakly positive $(+)$; 5-8 points, intermediately positive $(++)$; and 9 points and above, strongly positive $(+++) .+$ to +++ were marked as positive. Total positive rate $=$ (case of weakly positive + case of intermediately positive + case of strongly positive)/total case. 

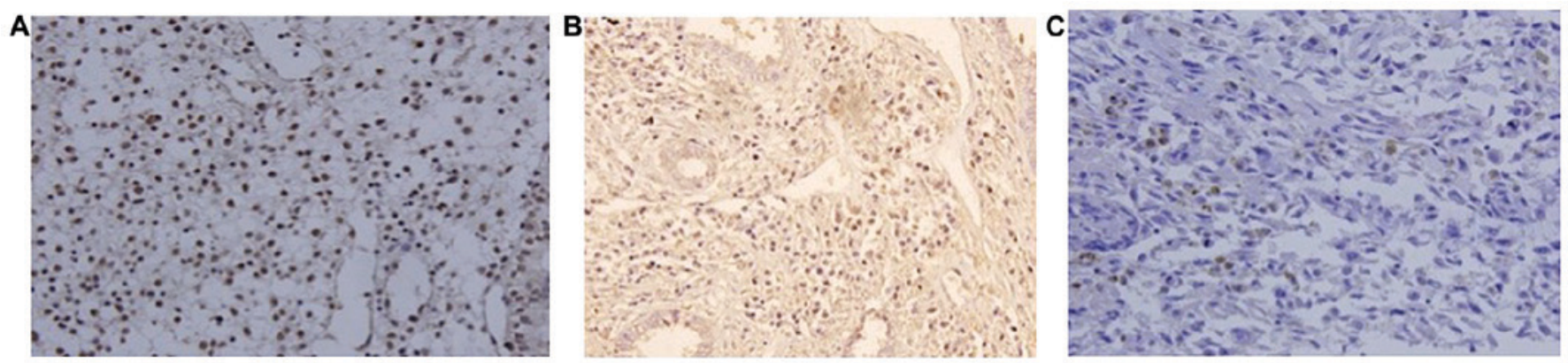

Figure 1. ELF5 is positively expressed in normal endometrial tissue. (A) ELF5 is positively expressed in normal endometrial tissue. (B) ELF5 is positively expressed in atypical hyperplasia of endometrium. (C) ELF5 is positively expressed in endometrial carcinoma tissue. ELF5, E74-like factor 5.

Postoperative follow-up of patients with endometrial carcinoma. Postoperative follow-up was conducted in all the patients with endometrial carcinoma. This included mainly outpatient follow-up and telephone interview. The follow-up started from the day when the patient underwent the surgery and ended June 30, 2017.

Statistical analysis. Statistical Product and Service Solutions (SPSS) 19.0 software (IBM Corp., Armonk, NY, USA) was utilized for statistical processing; $\chi^{2}$ test was used to examine the expression of ELF5 in different endometrial tissues. Kaplan-Meier method for survival analysis was applied for univariate analysis for evaluation of the effects of ELF5 and clinicopathologic parameters on the postoperative survival time of patients with endometrial carcinoma. Cox's proportional hazards regression model was utilized for univariate and multivariate analyses. The log-rank test was performed to examine the difference in survival rates between groups. $\mathrm{P}<0.05$ was considered to indicate a statistically significant difference.

\section{Results}

Comparisons of ELF5 expressions in different endometrial tissues (Table I). ELF5 was mainly expressed in nuclei and appeared as brownish yellow granules (Fig. 1). In normal endometrial tissue, atypical hyperplasia and endometrial carcinoma tissues, the positive expression rates of ELF5 were $93.33 \%$ $(28 / 30), 83.33 \%(25 / 30)$ and $69.05 \%$ (58/84), respectively. The above observation showed a decreasing tendency $\left(\chi^{2}=8.218\right.$, $\mathrm{P}=0.016)$. However, the statistically significant difference was observed only between normal group and endometrial carcinoma group $\left(\chi^{2}=5.787, \mathrm{P}=0.016\right)$. Furthermore, pairwise revealed non-significant results.

Relationship between ELF5 expressions and clinicopathologic parameters in endometrial carcinoma tissues (Table II). Relationship between ELF5 expressions and age. In the group aged $\leq 55$ years and the group aged $>55$ years, the positive expression rates of ELF5 were $70.73 \%$ (29/41) and $67.41 \%$ (29/43), respectively, but the difference was not statistically significant $\left(\chi^{2}=0.016, P=0.744\right)$. These age groups were selected to cover overall age of the patients affected with this disorder.

Relationship between ELF5 expressions and menstrual status. In premenopausal group and postmenopausal group, the positive expression rates of ELF5 were $66.67 \%(10 / 15)$ and $69.57 \%$ (48/69), respectively, but the difference was not statistically significant $\left(\chi^{2}=0.048, \mathrm{P}=0.826\right)$.

Relationship between ELF5 expression and FIGO staging. In phases I, II and III+IV, the positive expression rates of ELF5 were $94.44 \%$ (51/54), 40.00\% (4/10) and 15.00\% (3/20), respectively, and the differences among the three phases were statistically significant $\left(\chi^{2}=47.757, \mathrm{P}<0.05\right)$.

Relationship between ELF5 expression and pathological grading. According to the pathological grading and grouping, there were 68 cases of endometrial adenocarcinoma in total, including 50 cases of G1, 8 cases of G2 and 10 cases of G3; the positive expression rates of ELF5 were $94.00 \%$ (47/50), 37.5\% $(3 / 8)$ and $30 \%(3 / 10)$. All of the above differences among the three grades were statistically significant $\left(\chi^{2}=25.831, \mathrm{P}<0.05\right)$.

Relationship between ELF5 expression and lymph node metastasis. A total of 67 out of 84 patients with endometrial carcinoma underwent lymph node biopsy or lymphadenectomy. The positive expression rates of ELF5 in non-lymph node metastasis group and lymph node metastasis group were $92.68 \%$ (38/41) and $19.23 \%$ (5/26), respectively. The differences between the two groups were statistically significant $\left(\chi^{2}=34.212, \mathrm{P}<0.05\right)$.

Relationship between ELF5 expression and myometrial invasion. The positive expression rates of ELF5 in group with no myometrial invasion or with depth of myometrial invasion $\leq 1 / 2$ and group with depth of myometrial invasion $>1 / 2$ were $98.08 \%(51 / 52)$ and $21.88 \%$ (7/32), respectively. The differences between the two groups were statistically significant $\left(\chi^{2}=51.414, \mathrm{P}<0.05\right)$.

Relationship between ELF5 expressions and pathological types. The positive expression rates of ELF5 in endometrioid adenocarcinoma group and group with other pathological types were $77.94 \%(53 / 68)$ and $31.25 \%$ (5/16), respectively. The differences between the two groups were statistically significant $\left(\chi^{2}=11.118, \mathrm{P}=0.001\right)$.

Analysis on factors influencing prognosis of patients with endometrial carcinoma

Kaplan-Meier method for survival analysis (Table III).

Relationship between ELF5 expression and patient prognosis. A long-term follow-up for 6-67 months was conducted in 84 patients with endometrial carcinoma after operation, of which 18 patients were lost to follow-up and 11 patients died. The follow-up rate was $78.57 \%$. The mean survival time of 
Table II. Correlation of ELF5 expressions in endometrial carcinoma tissues with clinicopathological parameters.

\begin{tabular}{|c|c|c|c|c|c|c|}
\hline \multirow[b]{2}{*}{ Parameters } & \multirow[b]{2}{*}{ Total case (n) } & \multicolumn{2}{|c|}{ Expression } & \multirow[b]{2}{*}{ Positive rate (\%) } & \multirow[b]{2}{*}{$\chi^{2}$} & \multirow[b]{2}{*}{ P-value } \\
\hline & & Positive & Negative & & & \\
\hline \multicolumn{7}{|l|}{ Age (years) } \\
\hline$\leq 55$ & 41 & 12 & 29 & 70.73 & & \\
\hline$>55$ & 43 & 14 & 29 & 67.41 & 0.016 & 0.744 \\
\hline \multicolumn{7}{|l|}{ Menstruation } \\
\hline Pre-menopause & 15 & 5 & 10 & 66.67 & & \\
\hline Post-menopause & 69 & 21 & 48 & 69.57 & 0.048 & 0.826 \\
\hline \multicolumn{7}{|l|}{ Surgical-pathologic staging } \\
\hline I & 54 & 3 & 51 & 94.44 & & \\
\hline II & 10 & 6 & 4 & 40.00 & & \\
\hline III+IV & 20 & 17 & 3 & 15.00 & 47.757 & $<0.05$ \\
\hline \multicolumn{7}{|l|}{ Pathological grading ${ }^{a}$} \\
\hline G1 & 50 & 3 & 47 & 94.00 & & \\
\hline G2 & 8 & 5 & 3 & 37.50 & & \\
\hline G3 & 10 & 7 & 3 & 30.00 & 25.831 & $<0.05$ \\
\hline \multicolumn{7}{|l|}{ Lymph node metastasis ${ }^{\mathrm{b}}$} \\
\hline No & 41 & 3 & 38 & 92.68 & & \\
\hline Yes & 26 & 21 & 5 & 19.23 & 34.212 & $<0.05$ \\
\hline \multicolumn{7}{|l|}{ Myometrial invasion } \\
\hline No or $\leq 1 / 2$ & 52 & 1 & 51 & 98.08 & & \\
\hline$>1 / 2$ & 32 & 26 & 7 & 21.88 & 51.414 & $<0.05$ \\
\hline \multicolumn{7}{|l|}{ Pathological typing } \\
\hline Endometrioid adenocarcinoma & 68 & 15 & 53 & 77.94 & & \\
\hline Others & 16 & 11 & 5 & 31.25 & 11.118 & 0.001 \\
\hline
\end{tabular}

${ }^{\mathrm{a}} \mathrm{A}$ total of 68 out of 84 patients with endometrial carcinoma underwent pathological grading; ${ }^{\mathrm{b}} 67$ out of 84 patients with endometrial adenocarcinoma underwent lymph node biopsy or lymphadenectomy. ELF5, E74-like factor 5.

patients was 66.766 months in the negative ELF5 expression group, and it was 42.842 months in the positive ELF5 expression group. The overall survival time in the positive ELF5 expression group was higher $\left(\chi^{2}=8.508, \mathrm{P}=0.004\right)$ (Fig. 2).

Relationship between clinicopathological parameters and patients' prognosis. i) Age $\left(\chi^{2}=0.196, \mathrm{P}=0.658\right)$, menstrual status $\left(\chi^{2}=0.001, \mathrm{P}=0.975\right)$ or pathological type $\left(\chi^{2}=0.921\right.$, $\mathrm{P}=0.337$ ) revealed non-significant results.

ii) FIGO staging: the mean survival time in phases I, II and III+IV was 66.409, 50.571 and 41.067 months, respectively; the later the FIGO staging was, the poorer the prognosis would be; the differences among the three phases were statistically significant $\left(\chi^{2}=8.619, \mathrm{P}=0.011\right)$ (Fig. 3).

iii) Degree of myometrial invasion: the mean survival time in group with no myometrial invasion or with depth of myometrial invasion $\leq 1 / 2$ was 66.545 months, and it was 46.174 months in group with depth of myometrial invasion $>1 / 2$. The differences between the two groups were statistically significant $\left(\chi^{2}=5.109, \mathrm{P}=0.025\right)$ (Fig. 4).

iv) State of lymph node metastasis: the mean survival time in non-lymph node metastasis group was 66.545 months, and it was 40.550 months in lymph node metastasis group. The differences between the two groups were statistically significant $\left(\chi^{2}=5.380, P=0.020\right)$ (Fig. 5). v) Pathological grading: the mean survival time of patients with endometrial adenocarcinoma at G1, G2 and G3 was 65.850, 50.571 and 40.857 months, respectively. The higher the pathological grading was, the poorer the prognosis would be. The differences among the three grades were statistically significant $\left(\chi^{2}=6.093, \mathrm{P}=0.048\right)$ (Fig. 6).

Cox's regression model analyses. i) The univariate Cox's model analysis showed that age $\left(\chi^{2}=0.190, \mathrm{P}=0.663\right)$, pathological type $\left(\chi^{2}=0.870, \mathrm{P}=0.351\right)$ and menstrual status $\left(\chi^{2}=0.001, \mathrm{P}=0.975\right)$ were not associated with prognosis. On the other hand, FIGO staging $\left(\chi^{2}=6.619, \mathrm{P}=0.010\right)$, depth of myometrial invasion $\left(\chi^{2}=4.337, \mathrm{P}=0.037\right)$, lymph node metastasis $\left(\chi^{2}=4.788, \mathrm{P}=0.029\right)$, pathological grading $\left(\chi^{2}=4.225\right.$, $\mathrm{P}=0.040)$ and ELF5 $\left(\chi^{2}=6.706, \mathrm{P}=0.010\right)$ were related to prognosis (Table IV).

ii) Multivariate Cox's model analysis: the multivariate Cox's model analysis was performed on FIGO staging, depth of myometrial invasion, lymph node metastasis, pathological grading and ELF5 expression. The final screening results confirmed that ELF5 is an independent factor influencing the prognosis of patients with endometrial carcinoma $\left[\chi^{2}=4.424, \mathrm{P}=0.035\right.$; hazard ratio $(\mathrm{HR})=0.226$, 95\% CI: 0.056-0.904]. 
Table III. Kaplan-Meier method for survival analysis on prognosis of patients with endometrial carcinoma.

\begin{tabular}{|c|c|c|c|c|c|c|}
\hline Variables & Total case $(n)$ & Censored & $\begin{array}{l}\text { Mean survival } \\
\text { time (months) }\end{array}$ & $95 \% \mathrm{CI}$ & $\chi^{2}$ & P-value \\
\hline \multicolumn{7}{|l|}{ Age (years) } \\
\hline$\leq 55$ & 39 & 35 & 59.107 & $51.864-66.350$ & \multirow[t]{2}{*}{0.196} & \multirow[t]{2}{*}{0.658} \\
\hline$>55$ & 45 & 38 & 60.447 & $52.699-68.195$ & & \\
\hline \multicolumn{7}{|l|}{ Menstruation } \\
\hline Pre-menopause & 69 & 60 & 57.087 & $44.494-69.673$ & \multirow[t]{2}{*}{0.001} & \multirow[t]{2}{*}{0.975} \\
\hline Post-menopause & 15 & 13 & 61.796 & $55.678-67.915$ & & \\
\hline \multicolumn{7}{|l|}{ Surgical-pathologic staging } \\
\hline I & 54 & 50 & 66.409 & $61.180-71.638$ & \multirow[t]{3}{*}{8.169} & \multirow[t]{3}{*}{0.004} \\
\hline II & 10 & 9 & 50.571 & $38.906-62.237$ & & \\
\hline III+IV & 20 & 14 & 41.067 & $27.887-54.256$ & & \\
\hline \multicolumn{7}{|l|}{ Pathological grading } \\
\hline $\mathrm{G} 1$ & 50 & 47 & 65.850 & $60.127-71.573$ & \multirow[t]{3}{*}{6.093} & \multirow[t]{3}{*}{0.048} \\
\hline G2 & 8 & 7 & 50.571 & $38.906-62.327$ & & \\
\hline G3 & 10 & 7 & 40.857 & $22.354-59.360$ & & \\
\hline \multicolumn{7}{|l|}{ Lymph node metastasis } \\
\hline No & 41 & 38 & 66.545 & $60.660-72.431$ & \multirow[t]{2}{*}{5.380} & \multirow[t]{2}{*}{0.020} \\
\hline Yes & 26 & 19 & 40.550 & $30.563-50.537$ & & \\
\hline \multicolumn{7}{|l|}{ Myometrial invasion } \\
\hline No or $\leq 1 / 2$ & 52 & 48 & 66.279 & $60.934-71.624$ & \multirow[t]{2}{*}{5.109} & \multirow[t]{2}{*}{0.024} \\
\hline$>1 / 2$ & 32 & 25 & 46.174 & $36.372-56.076$ & & \\
\hline \multicolumn{7}{|l|}{ Pathological typing } \\
\hline Endometrioid adenocarcinoma & 68 & 60 & 63.037 & $57.278-68.796$ & \multirow[t]{2}{*}{0.921} & \multirow[t]{2}{*}{0.337} \\
\hline Others & 16 & 13 & 48.167 & $34.531-61.802$ & & \\
\hline \multicolumn{7}{|l|}{ ELF5 } \\
\hline Positive & 58 & 54 & 66.766 & $61.855-71.677$ & \multirow[t]{2}{*}{8.508} & \multirow[t]{2}{*}{0.004} \\
\hline Negative & 26 & 19 & 42.842 & $31.407-54.278$ & & \\
\hline
\end{tabular}

CI, confidence interval; ELF5, E74-like factor 5.

Table IV. Analysis results of Cox's proportional hazards regression model in patients with endometrial carcinoma.

\begin{tabular}{lccccccc}
\hline & & & & & \multicolumn{2}{c}{$95 \%$ CI } \\
\cline { 5 - 7 } Variables & Regression coefficient & Standard error & Wald $\chi^{2}$ & P-value & Exp (B) & Lower limit & Upper limit \\
\hline FIGO staging & 0.848 & 0.330 & 6.619 & 0.010 & 2.335 & 1.224 \\
Myometrial invasion & 1.306 & 0.627 & 4.337 & 0.037 & 3.691 & 1.080 & 12.617 \\
Lymph node metastasis & 1.511 & 0.691 & 4.788 & 0.029 & 4.532 & 1.171 \\
ELF5 expression & -1.625 & 0.627 & 6.706 & 0.010 & 0.197 & 0.058 & 0.674 \\
Age & -0.137 & 0.313 & 0.190 & 0.663 & 0.872 & 0.472 & 1.612 \\
Pathological type & -0.316 & 0.339 & 0.870 & 0.351 & 0.729 & 0.375 & 1.416 \\
Menstruation & -0.012 & 0.391 & 0.001 & 0.975 & 0.988 & 0.459 & 2.125 \\
Pathological grading & 0.815 & 0.397 & 4.225 & 0.040 & 2.269 & 1.039 \\
\hline
\end{tabular}

FIGO, International Federation of Gynecology and Obstetrics; CI, confidence interval; ELF5, E74-like factor 5.

\section{Discussion}

Members of the ETS family play a vital regulatory role in normal physiological activities, such as development, differentiation, proliferation and apoptosis of cells (7). An earlier study reported that the expression of ETS-related proteins was upregulated in renal cell carcinoma in comparison to normal kidney (15). ELF5, also known as ESE2, is a member of the ETS transcription factor family. It has two isomers, namely ESE2a and ESE2b that exist in human body as a result of 


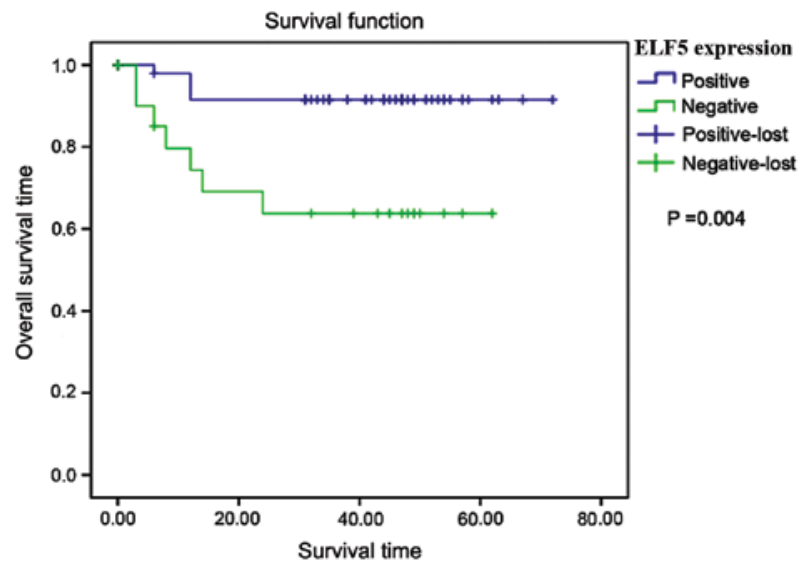

Figure 2. Relationship between ELF5 expression in endometrial carcinoma tissues and overall survival. ELF5, E74-like factor 5.

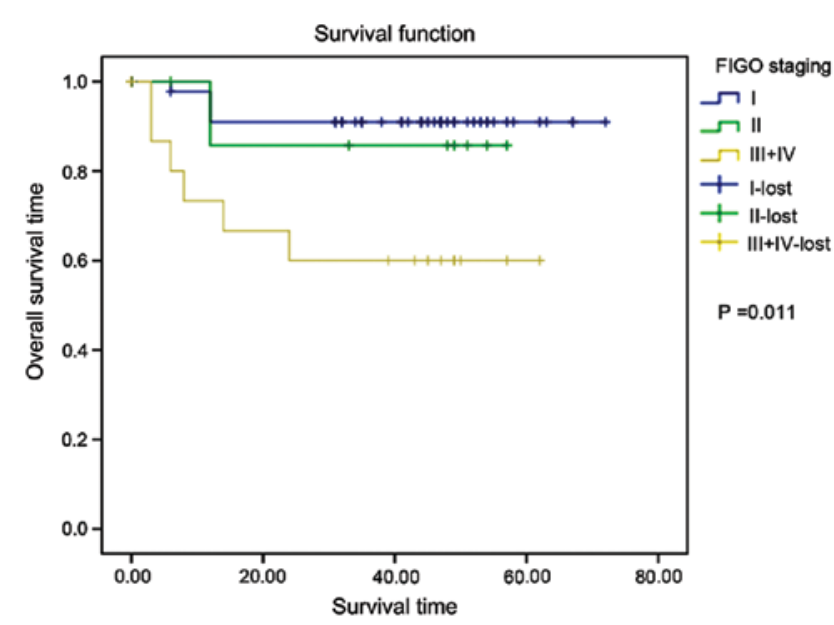

Figure 3. Relationship between FIGO staging and overall survival in patients with endometrial carcinoma. FIGO, International Federation of Gynecology and Obstetrics.

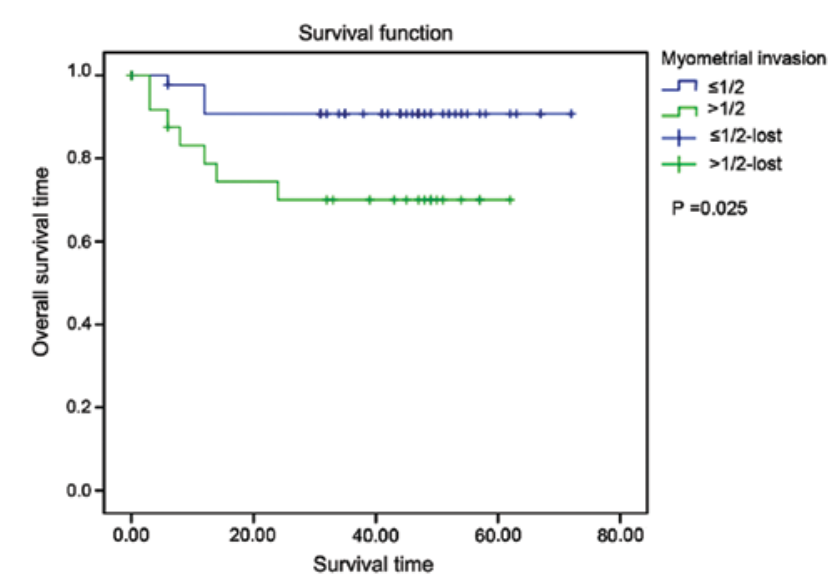

Figure 4. Relationship between degree of myometrial invasion and overall survival in patients with endometrial carcinoma.

alternate transcriptional start sites localized in distinctive exon 1s. Moreover, ELF5 has epithelial specificity and is expressed in placenta, lung airway, mammary gland, kidney and other organs $(7,10)$.

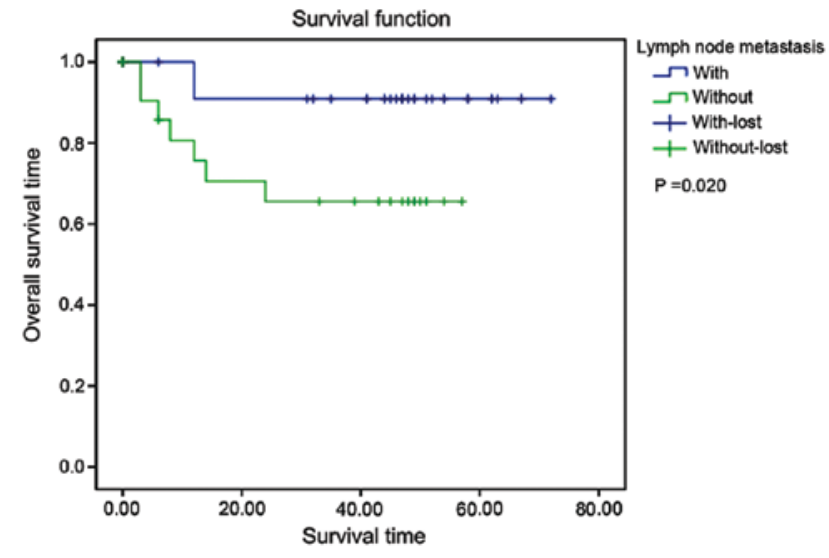

Figure 5. Relationship between state of lymph node metastasis and overall survival in patients with endometrial carcinoma.

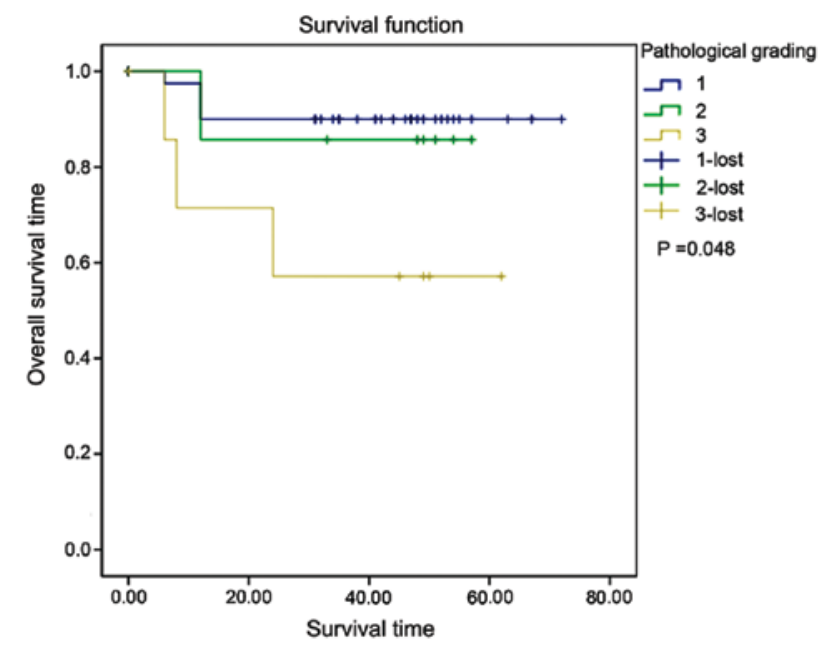

Figure 6. Relationship between pathological grading and overall survival in patients with endometrial carcinoma.

In research related to ELF5 and breast cancer, researchers have observed loss of ELF5 expression in tissues and cell lines of human breast cancer as a potential suppressor gene (10). In the present study, ELF5 expression in normal endometrial tissue, atypical hyperplasia of endometrium and endometrial carcinoma tissues, showed a decreasing tendency. Further, the group wise comparison revealed non-significant results due to the small sample size of the present study. Further, the immunohistochemical and statistical results showed that reduced or absent expression of ELF5 might have promoted the occurrence of endometrial carcinoma. Yet the relevant mechanism has not been determined. Moreover, it is quite possible that, like that of other ETS factors, the abnormal expression in cancers leads to dysregulation of target genes, thus causing cell transformation.

An earlier study confirmed that the expression of ELF5 mRNA had a negative correlation with staging and lymph node metastasis, but it was not associated with age, pathological grading, tissue type and ascites (12). Similarly in the present study, the positive expression rate of ELF5 in endometrial carcinoma tissue showed decrease. The study also showed the increase of FIGO staging, pathological grading, 
depth of myometrial invasion and progression of lymph node metastasis. However, it was not related to age and menopause. This confirmed that the changes in ELF5 expression have a correlation with tumor development. The ELF5 showed a close association with the staging of endometrial carcinoma in an earlier study (16). This might be related to the fact that ELF5 could inhibit the occurrence of epithelial-mesenchymal transition (EMT). Some studies showed that ELF5 not only acts as a crucial regulator of cell lineage, but also suppresses the occurrence of EMT by inhibition of the transcription of Snail2 (17). EMT affects cell adhesion, polarity and migration by means of changing the state of epithelial cells to mesenchymal stem cells. In tumor cells, EMT and its reverse program were activated so that the tumor cells could acquire higher-level of invasiveness and features related to relapse (18). It could be concluded that the decrease or loss of ELF5 expression in endometrial carcinoma could weaken the inhibition to EMT, thereby, accelerated the development of endometrial carcinoma.

The expression of ELF5 is closely associated with hormones. In mammary gland, prolactin and progesterone can promote the expression of ELF5, which could accelerate the development of embryonic precursor cells into estrogen receptor $\alpha$ and progesterone receptors (19). Type I endometrial carcinoma is estrogen-dependent, and its mechanism is a result of long-term effect of estrogen without antagonism of progesterone (20). The results of this research showed that the positive expression rate of ELF5 in endometrioid adenocarcinoma was higher than that of other pathological types (such as serous carcinoma and squamous cell carcinoma). The association of ELF5 with estrogen, progesterone and their receptors, as well as its molecular mechanism, needs to be determined by further studies.

There are many influencing factors for the prognosis of patients with endometrial carcinoma. Boks et al (21) revealed by univariate survival analysis that age, menopause, vaginal bleeding, pathological type, histological grading, surgical-pathologic staging, degree of myometrial invasion, lymph node metastasis, positive peritoneal cytology and postoperative adjunctive therapy have effects on the prognosis of endometrial carcinoma. Biological factors related to prognosis of cancers have attracted the attention of the researchers (22); for instance, multiple studies have indicated that the expression levels of ER and progesterone receptors (PR) are important factors for judging the prognosis of patients with endometrial carcinoma. In the present study, the univariate survival analysis revealed that surgical-pathologic staging, histological grading, depth of myometrial invasion, lymph node metastasis and ELF5 were the factors affecting the prognosis of endometrial carcinoma. Multivariate Cox regression model analysis showed that ELF5 was an independent factor influencing endometrial carcinoma. The mortality risk of patients with positive ELF5 in endometrial carcinoma was decreased in comparison with the patients with negative ELF5 (HR $=0.226,95 \%$ CI: 0.056-0.904). Moreover, the mortality risk of patients with positive ELF5 was 0.226 times as high as that of patients with negative ELF5. So, ELF5 could be utilized as an ideal biological parameter for judging the prognosis of endometrial carcinoma. The number of follow-up cases was small, of which 12 cases had a follow-up time longer than
5 years (including 5 years) in the present study. Therefore, it would be more persuasive if the number of follow-up cases was more and the follow-up time was extended.

In conclusion, ELF5 could be used as an independent impact factor for evaluating the prognosis of endometrial carcinoma. It has good prospects for clinical application in early screening and prognosis evaluation of endometrial carcinoma but future studies with large sample sizes are essential for concrete conclusion.

\section{Acknowledgements}

Not applicable.

\section{Funding}

No funding was received.

\section{Availability of data and materials}

The datasets used and/or analyzed during the present study are available from the corresponding author on reasonable request.

\section{Authors' contributions}

HY collected and analyzed the general information of patients. HY and HCY were responsible for IHC and follow-up analysis. Both authors read and approved the final manuscript.

\section{Ethics approval and consent to participate}

The Ethics Committee of the Affiliated Hospital of Xuzhou Medical University (Jiangsu, China) approved the study and written informed consents were signed by the patients and/or guardians.

\section{Patient consent for publication}

Not applicable.

\section{Competing interests}

The authors declare that they have no competing interests.

\section{References}

1. Morice P, Leary A, Creutzberg C, Abu-Rustum N and Darai E: Endometrial cancer. Lancet 387: 1094-1108, 2016.

2. Siegel R, Naishadham D and Jemal A: Cancer statistics, 2013. CA Cancer J Clin 63: 11-30, 2013.

3. Stewart CJ, Doherty DA, Havlat M, Koay MH, Leung YC, Naran A, O'Brien D, Ruba S, Salfinger S and Tan J: Transtubal spread of endometrial carcinoma: Correlation of intra-luminal tumour cells with tumour grade, peritoneal fluid cytology, and extra-uterine metastasis. Pathology 45: 382-387, 2013.

4. Maritschnegg E, Wang Y, Pecha N, Horvat R, Van Nieuwenhuysen E, Vergote I, Heitz F, Sehouli J, Kinde I, Diaz LA Jr, et al: Lavage of the uterine cavity for molecular detection of Müllerian duct carcinomas: A Proof-of-Concept Study. J Clin Oncol 33: 4293-4300, 2015

5. Benedetti Panici P, Basile S, Salerno MG, Di Donato V, Marchetti C, Perniola G, Palagiano A, Perutelli A, Maneschi F, Lissoni AA, et al: Secondary analyses from a randomized clinical trial: age as the key prognostic factor in endometrial carcinoma. Am J Obstet Gynecol 210: 363.e1-363.e10, 2014. 
6. Abu-Rustum NR1, Iasonos A, Zhou Q, Oke E, Soslow RA, Alektiar KM, Chi DS and Barakat RR: Is there a therapeutic impact to regional lymphadenectomy in the surgical treatment of endometrial carcinoma? Am J Obstet Gynecol 198: 457.e1-e6, 2008.

7. Lee HJ and Ormandy CJ: Elf5, hormones and cell fate. Trends Endocrinol Metab 23: 292-298, 2012.

8. Lee HJ, Gallego-Ortega D, Ledger A, Schramek D, Joshi P, Szwarc MM, Cho C, Lydon JP, Khokha R, Penninger JM, et al: Progesterone drives mammary secretory differentiation via RankL-mediated induction of Elf5 in luminal progenitor cells. Development 140: 1397-1401, 2013.

9. Kalyuga M, Gallego-Ortega D, Lee HJ, Roden DL, Cowley MJ, Caldon CE, Stone A, Allerdice SL, Valdes-Mora F, Launchbury R, et al: ELF5 suppresses estrogen sensitivity and underpins the acquisition of antiestrogen resistance in luminal breast cancer. PLoS Biol 10: e1001461, 2012.

10. Pearton DJ, Smith CS, Redgate E, van Leeuwen J, Donnison M and Pfeffer PL: Elf5 counteracts precocious trophoblast differentiation by maintaining Sox 2 and 3 and inhibiting Hand 1 expression. Dev Biol 392: 344-357, 2014.

11. Chakrabarti R, Wei Y, Romano RA, DeCoste C, Kang Y and Sinha S: Elf5 regulates mammary gland stem/progenitor cell fate by influencing notch signaling. Stem Cells 30: 1496-1508, 2012.

12. Yan H, Qiu L, Xie X, Yang H, Liu Y, Lin X and Huang H: ELF5 in epithelial ovarian carcinoma tissues and biological behavior in ovarian carcinoma cells. Oncol Rep 37: 1412-1418, 2017.

13. Endo A, Tomizawa D, Aoki Y, Morio T, Mizutani S and Takagi M: EWSR1/ELF5 induces acute myeloid leukemia by inhibiting p53/p21 pathway. Cancer Sci 107: 1745-1754, 2016

14. Yan H, Qiu L, Xie X, Yang H, Liu Y, Lin X and Huang H: ELF5 in epithelial ovarian carcinoma tissues and biological behavior in ovarian carcinoma cells. Oncol Rep 37: 1412-1418, 2017.

15. Grassmeyer J, Mukherjee M, deRiso J, Hettinger C, Bailey M, Sinha S, Visvader JE, Zhao H, Fogarty E and Surendran K: Elf5 is a principal cell lineage specific transcription factor in the kidney that contributes to Aqp2 and Avpr2 gene expression. Dev Biol 424: 77-89, 2017.
16. Risinger JI, Maxwell GL, Chandramouli GV, Jazaeri A, Aprelikova O, Patterson T, Berchuck A and Barrett JC: Microarray analysis reveals distinct gene expression profiles among different histologic types of endometrial cancer. Cancer Res 63: 6-11, 2003.

17. Chakrabarti R, Hwang J, Andres Blanco M, Wei Y,Lukačišin M, Romano RA, Smalley K, Liu S, Yang Q, Ibrahim T, et al: Elf5 inhibits the epithelial-mesenchymal transition in mammary gland development and breast cancer metastasis by transcriptionally repressing Snail2. Nat Cell Biol 14: 1212-1222, 2012

18. Zhou ZJ, Dai Z, Zhou SL, Hu ZQ, Chen Q, Zhao YM, Shi YH, Gao Q, Wu WZ, Qiu SJ, et al: HNRNPAB induces epithelial-mesenchymal transition and promotes metastasis of hepatocellular carcinoma by transcriptionally activating SNAIL. Cancer Res 74: 2750-2762, 2014.

19. Oakes SR, Naylor MJ, Asselin-Labat ML, Blazek KD, Gardiner-Garden M, Hilton HN, Kazlauskas M, Pritchard MA, Chodosh LA, Pfeffer PL, et al: The Ets transcription factor Elf5 specifies mammary alveolar cell fate. Genes Dev 22: 581-586, 2008.

20. Kreizman-Shefer H, Pricop J, Goldman S, Elmalah I and Shalev E: Distribution of estrogen and progesterone receptors isoforms in endometrial cancer. Diagn Pathol 9: 77, 2014.

21. Boks DE, Trujillo AP, Voogd AC, Morreau H, Kenter GG and Vasen HF: Survival analysis of endometrial carcinoma associated with hereditary nonpolyposis colorectal cancer. Int J Cancer 102: 198-200, 2002.

22. Dong P, Kaneuchi M, Konno Y, Watari H, Sudo S and Sakuragi N: Emerging therapeutic biomarkers in endometrial cancer. BioMed Res Int 2013: 130362, 2013

(7) This work is licensed under a Creative Commons EY No NO Attribution-NonCommercial-NoDerivatives 4.0 International (CC BY-NC-ND 4.0) License. 